\title{
Temperature window effect and its application in extrusion of ultrahigh molecular weight polyethylene
}

\author{
L. M. Fang ${ }^{1,2^{*}}$, P. Gao ${ }^{3}$, X. W. $\mathrm{Cao}^{2}$ \\ ${ }^{1}$ Biomedical Engineering Institute, South China University of Technology, Guangzhou 510640, China \\ ${ }^{2}$ Key Laboratory of Polymer Processing Engineering, Ministry of Education, South China University of Technology, \\ Guangzhou 510640, China \\ ${ }^{3}$ Department of Chemical and Biomolecular Engineering, The Hong Kong University of Science and Technology, Clear \\ Water Bay, Kowloon, Hong Kong
}

\begin{abstract}
Ultrahigh molecular weight polyethylene (UHMWPE) was ram extruded using a temperature window effect. The extrusion pressure abruptly drops at a very narrow extrusion temperature window which is about $10^{\circ} \mathrm{C}$ higher than the theoretical melting point of orthorhombic polyethylene crystals under quiescent and equilibrium states. The correlation between extrusion pressure and parameters such as extrusion temperature, annealing condition, thermal history, piston velocity, $L / D$ ratio of the die, and molecular weight of UHMWPE, was studied. The temperature window increases with molecular weight and is unaffected by thermal history and annealing. The stable extrusion pressure and the critical piston velocity decrease with the rise in the extrusion temperature. The flow resistance reversely depends on the $L / D$ ratio of the die. This phenomenon is attributed to an extensional flow-induced chain alignment along the streamline, which results in the formation of a metastable mesophase with higher chain mobility.
\end{abstract}

Keywords: biocompatible polymers, processing technologies, temperature window, UHMWPE

\section{Introduction}

Ultrahigh molecular weight polyethylene (UHMWPE) exhibits extraordinary properties, such as wear and impact resistance, which make the material ideal for application as joint prostheses and bullet-proof vests $[1,2]$. However, its extremely high melt viscosity makes processing via conventional techniques, such as screw extrusion or injection molding, difficult [3].

Problems that are commonly encountered in processing UHMWPEs include die blockage, melt fracture, wall slippage, and a small processing temperature window. Processing UHMWPE, therefore, requires a proper combination of temperature, pressure, and sufficient time to achieve complete plastification. Inadequate control of the process can lead to fusion defects stemming from the memory of the powder morphology [1, 4]. Currently, UHMWPE resin is consolidated mainly by compression molding or ram extrusion [3]. Compression molding progresses slowly and involves costly equipment, whereas ram extrusion is relatively cheap. However, some unconsolidated regions in the center of the material may occur. Considerable efforts have been devoted to developing better methods for UHMWPE processing.

Since the discovery of the extended-chain hexagonal crystal phase of polyethylene in the 1960s [5, 6], the enhanced chain mobility of this phase has elicited numerous theoretical and experimental investigations. However, the extremely high pressure $(>360 \mathrm{MPa})$ and temperature $\left(>230^{\circ} \mathrm{C}\right)$ associ-

\footnotetext{
${ }^{*}$ Corresponding author, e-mail: 1mfang@scut.edu.cn

(c) BME-PT
} 
ated with the formation of hexagonal crystals make processing in this phase almost impossible. The crystallization of chain segments immediately after synthesis reduces the likelihood of entanglement formation and favors more extended chain conformations; thus, nascent reactor powders have been suggested to possess low degrees of entanglement $[7,8]$. Because of this, solid-state extrusion or cold extrusion was developed. However, the extrudate needs to be drawn into fibers or films after cold extrusion [9].

In 1990, Waddon and Keller [10] observed a strange phenomenon during capillary rheological tests of medium high molecular weight polyethylene (MHMWPE). In a narrow temperature interval (150$152^{\circ} \mathrm{C}$ ), a minimum melt flow resistance was discovered. Keller and his associates called this phenomenon 'temperature window effect' and systematically investigated it in a series of papers [11-13] and in a review [14]. Keller and coworkers [15-22], Cheng [23] and Somani et al. [25] independently or collaboratively studied the mechanisms of unexpected melt flow behavior, and attributed reduced flow resistance to the transient mobile hexagonal mesophase by flow-induced chain alignment critical to strain rate and molecular weight. This hypothesis was confirmed by the observation of the orthorhombic transformation to the hexagonal phase through in situ wide angle X-ray diffraction [25-27].

These new findings have potential significance for polymer processing at low pressures. However, the window effect is strongly dependent on molecular weight. Kolnaar and Keller [11] defined molecular weight dependence within the range of $1.3 \cdot 10^{5}$ to $1.0 \cdot 10^{6} \mathrm{~g} / \mathrm{mol}$. To process UHMWPE in the temperature window, Narh and Keller [28] dissolved nascent UHMWPE in a solvent, extruded the dried gel, and obtained a pronounced pressure minimum at which a smooth extrudate was observed. However, a large amount of toxic solvent, usually decalin or xylene, is employed in this process. A solution-free route is preferred. Rastogi et al. [27] studied the orthorhombic-hexagonal phase transition of solution-crystallized UHMWPE by in situ small-angle X-ray scattering at pressures of 160 and $180 \mathrm{MPa}$ during heating or cooling. The experiments revealed that the location of the triple point in the pressure-temperature phase diagram of polyethylene depended on crystal dimensions, that is, fold length. Based on the results, a UHMWPE sheet was compression-molded in the hexagonal phase at $120 \mathrm{MPa}$ and $205^{\circ} \mathrm{C}$, and then subsequently melted and recrystallized isobarically. The fusion defects substantially decreased, but the processing pressure and temperature were still excessively high.

Previous studies have indicated that the temperature window effect depends on hexagonal phase stability, which in turn, depends on the alignment of polymer chains [15-17, 22, 29]. Compared with MHMWPE, UHMWPE chains are much longer. Accordingly, disentanglement and alignment of UHMWPE chains are much more difficult. Therefore, the key is to induce the mobile hexagonal phase by an elongational flow field and then maintain its stability. The aim of this paper is to study influences of various factors, including extrusion temperature, annealing condition, thermal history, piston velocity, $L / D$ ratio of the die, and molecular weight of UHMWPE, on the temperature window effect, mainly from the processing viewpoint.

\section{Experimental}

\subsection{Materials and equipment}

Three UHMWPE resins were used. M-II and M-III, with molecular weights $\left(M_{\mathrm{w}}\right) 2.5$ and 3.5 million $\mathrm{g} / \mathrm{mol}$, respectively, were purchased from Beijing Eastern Petrochemical Co., Ltd (Beijing, China). GUR 1050, with an $M_{\mathrm{w}}$ of 4.9 million $\mathrm{g} / \mathrm{mol}$, was supplied by Ticona (Celanese Co., Ltd., Singapore). A high pressure capillary rheometer (Rheologic 5000-Twin, CEAST S.P.A., Pianezza, Italy) was used to process UHMWPE rods. The barrel length and diameter are 300 and $15 \mathrm{~mm}$, respectively. The temperature was controlled in the range of $50-450^{\circ} \mathrm{C}$ with an accuracy of $0.5^{\circ} \mathrm{C}$. The working ranges of the force transducer, pressure transducer, and piston velocity were $5-40 \mathrm{kN}, 3.5-200 \mathrm{MPa}$, and $0.01-$ $1000 \mathrm{~mm} / \mathrm{min}$, respectively. VisualRHEO software (CEAST S.P.A., Pianezza, Italy) was used to control the extrusion process and analyze the processing parameters.

Convergent cylindrical dies with an entrance angle of $90^{\circ}$, length/diameter $(L / D)$ ratio of 10:5, 15:5, and $18: 5[\mathrm{~mm} / \mathrm{mm}]$, and total length of $40 \mathrm{~mm}$ were designed (Figure 1). A part of the screw was machined to the bottom of the self-made hardened stainless steel die, and a bolt was used to prevent the powder from leaking during charging. 


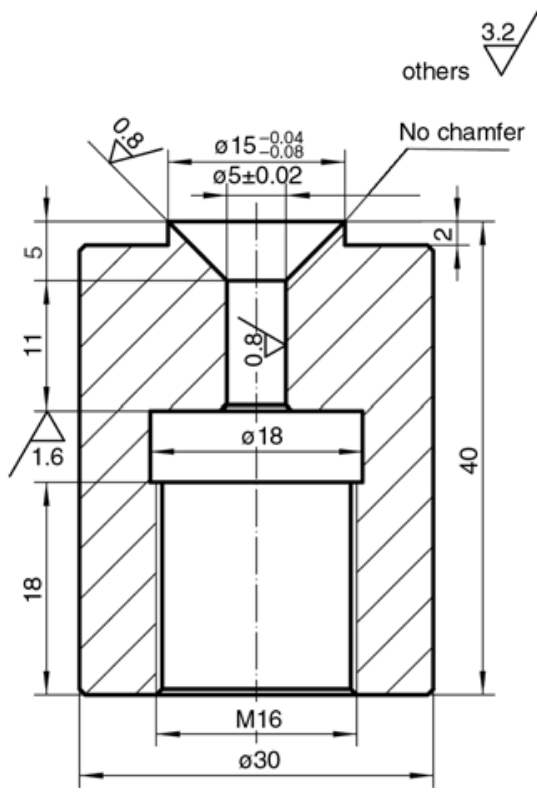

Figure 1. Typical design drawing of the extrusion die $(L / D$ ratio: $10: 5, \mathrm{~mm} / \mathrm{mm}$ )

\subsection{Extrusion procedures}

The extrusion steps are listed as follows: 1) The entire system, including the piston, barrel, and die, was carefully cleaned because surface quality influences extrusion pressure. 2) The system was setup with proper alignment and tight contact of parts to reduce friction and ensure that the melt is not expelled from the gap. 3) The barrel was intermittently charged with powder and then compressed to obtain densely packed particles. 4) Gas/moisture trapped in the nodular pores was released by degassing at $120^{\circ} \mathrm{C}$. 5) The resin was melted at a given temperature and pressure at specific periods (30, 60, and $90 \mathrm{~min}) .6$ ) The melt was cooled to the extrusion temperature window. 7) The temperature was conditioned for about $15 \mathrm{~min}$ to reach equilib- rium and avoid the temperature gradient between the wall and the center. 8) The block was uninstalled and the extrusion carried out at a preset piston velocity.

Majority of the experiments consisted of measuring pressure $(p)$ as a function of temperature $(T)$ at a constant piston velocity $(v)$. Pressure traces were recorded as a function of time at a fixed piston velocity while the barrel temperature was kept constant. Various parameters, including extrusion temperature, annealing process, thermal history, piston velocity, $L / D$ ratio of the die, and molecular weight, were investigated during UHMWPE processing via the metastable phase.

\section{Results and discussion}

\subsection{Effects of temperature}

The resin (M II) was melted at $200^{\circ} \mathrm{C}$ for $1 \mathrm{~h}$ under $45 \mathrm{MPa}$; the temperature was then decreased to $145^{\circ} \mathrm{C}$ and the pressure was decreased to around $2 \mathrm{MPa}$. After being conditioned for $15 \mathrm{~min}$, the melt was extruded at a speed of $0.035 \mathrm{~mm} / \mathrm{s}$. Pressure traces were recorded as a function of time (Figure 2). Figure 2 indicates that extrusion pressure linearly increases with extrusion time, and then abruptly decreases after $50-160$ seconds in the temperature range of $154-157^{\circ} \mathrm{C}$. This temperature range is defined as the temperature window and the phenomenon is called the temperature window effect [10]. If extrusion temperature is below this window, the pressure continuously increases to the maximum working range of the transducer; thus, the rod cannot be extruded. If the extrusion temperature is higher than the window, pressure begins to fluctuate and the rod becomes distorted. Higher tem-
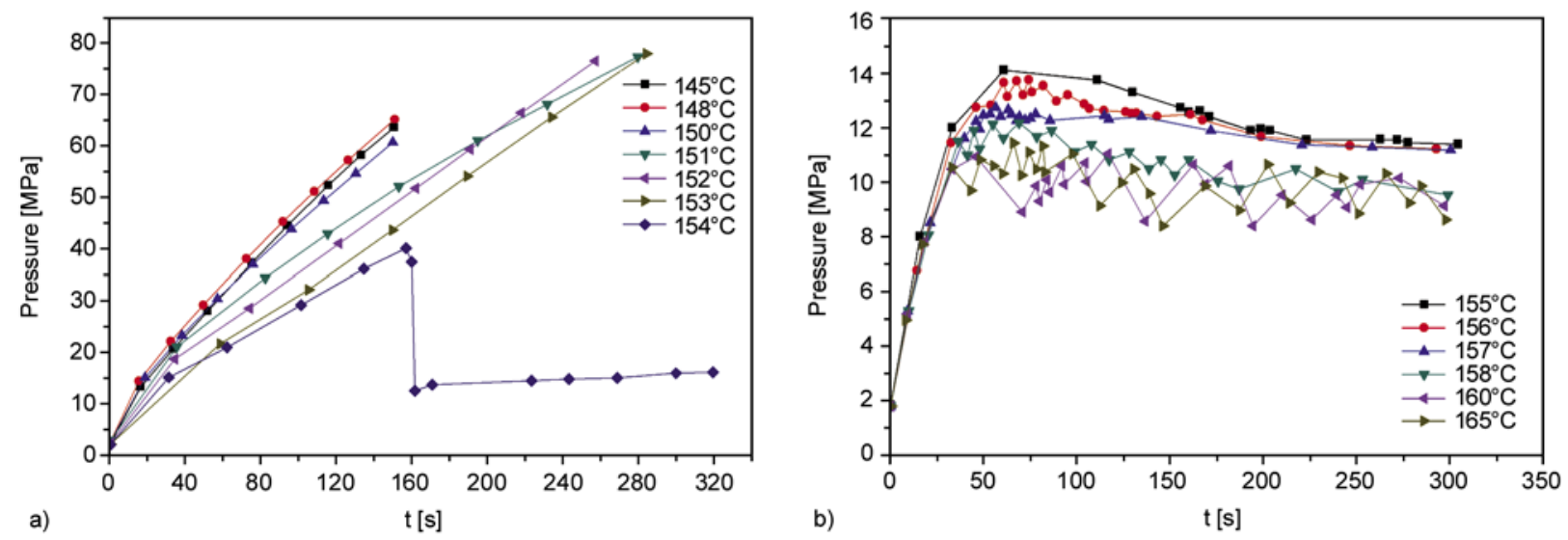

Figure 2. Time evolution of extrusion pressure at different extrusion temperatures $\left(M_{\mathrm{w}}=3.5 \cdot 10^{6} \mathrm{~g} / \mathrm{mol} ; L / D=10: 5\right.$, $\mathrm{mm} / \mathrm{mm} ; v=0.035 \mathrm{~mm} / \mathrm{s}$ ) 
perature brings about more severe fluctuation, implying that the stable extrusion of the material can only be performed within the narrow temperature window.

Below the temperature window, the UHMWPE melt exhibits elastic behavior. Flow activation energy is too high to generate flow of the entangled melt, causing the extrusion pressure increases with the extrusion time. The work done by the compression of the piston is stored in the melt. With the increase of extrusion temperature, the chain mobility in UHMWPE melt is enhanced, leading to the decrease of extrusion pressure.

When the extrusion temperature is increased to $154^{\circ} \mathrm{C}$, the melt is compressed at the initial stage and flows like rubber for about $150 \mathrm{~s}$. The melt flows from the entry of the convergent die $(\sim 5.0 \mathrm{~mm})$ and enters the 'capillary'. The extent of chain extension differs from the center to the wall. The elastic energy stored in the melt during flow in the convergent entry of the die is released abruptly when the melt enters the capillary, thereby reducing extrusion pressure. The melt flows steadily at a constant extrusion speed - a phenomenon that can be explained from two aspects: (1) chain mobility is enhanced at temperatures $10^{\circ} \mathrm{C}$ higher than the theoretical equilibrium melting point of orthorhombic polyethylene crystals [30]; and (2) chains are extended at the orifice and aligned along the flow direction, producing a chain-extended structure similar to the hexagonal crystal phase [26]. This is the so-called elongational flow-induced crystallization [11]. With the increase in extrusion temperature, the flow activation energy declines, and the amplitude of the pressure drop is

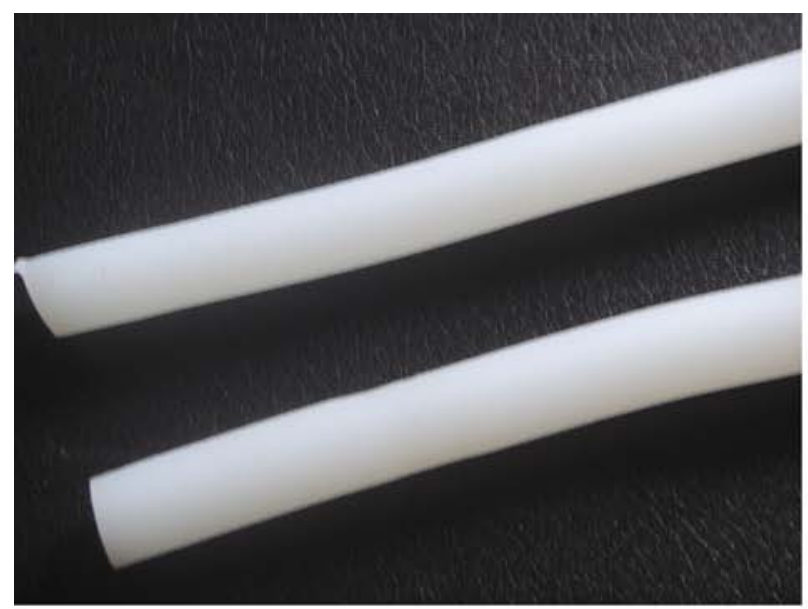

a)

Figure 3. Photos of rods extruded at different temperatures $\left(M_{\mathrm{w}}=3.5 \cdot 10^{6} \mathrm{~g} / \mathrm{mol} ; L / D=10: 5, \mathrm{~mm} / \mathrm{mm} ; v=0.035 \mathrm{~mm} / \mathrm{s}\right)$. a) In the temperature window $\left(T=154^{\circ} \mathrm{C}\right)$; b) above the temperature window $\left(T=165^{\circ} \mathrm{C}\right)$ of lower magnitude. However, in all the experiments, the pressure stabilizes at around $12 \mathrm{MPa}$ in extrudate with a smooth surface (Figure $3 a$ ).

After the temperature is increased to $158^{\circ} \mathrm{C}$, extrusion becomes unstable immediately after the initial stage. The extended chain structure induced by elongational flow is a non-equilibrium state, and this transient phase is metastable in a narrow temperature range under shear [14]. Non-equilibriumextended chains transform into entangled chains, achieving an equilibrium state. Chain entanglement in the melt becomes severe at high temperature, and critical shear rate is very small, resulting in melt fracture (Figure 3b). With the increase in extrusion temperature, the pressure fluctuation becomes violent, and the extrudate surface becomes severely distorted, as revealed by the fluctuation amplitude and frequency.

Further increasing the temperature to above $200^{\circ} \mathrm{C}$, which is the temperature range for traditional ram extrusion, makes for successful processing. However, the extrusion speed is slower and the extrusion pressure is much higher compared with those in the temperature window $154-157^{\circ} \mathrm{C}$ [1].

\subsection{Effects of annealing process}

Kolnaar and Keller [11] reported that the resin should be annealed sufficiently to erase the nascent structure before extrusion in the temperature window. To study the influence of annealing process on the temperature window effect during the extrusion of UHMWPE, we melted the resin at different temperatures $\left(180\right.$ or $\left.200^{\circ} \mathrm{C}\right)$ for certain time periods

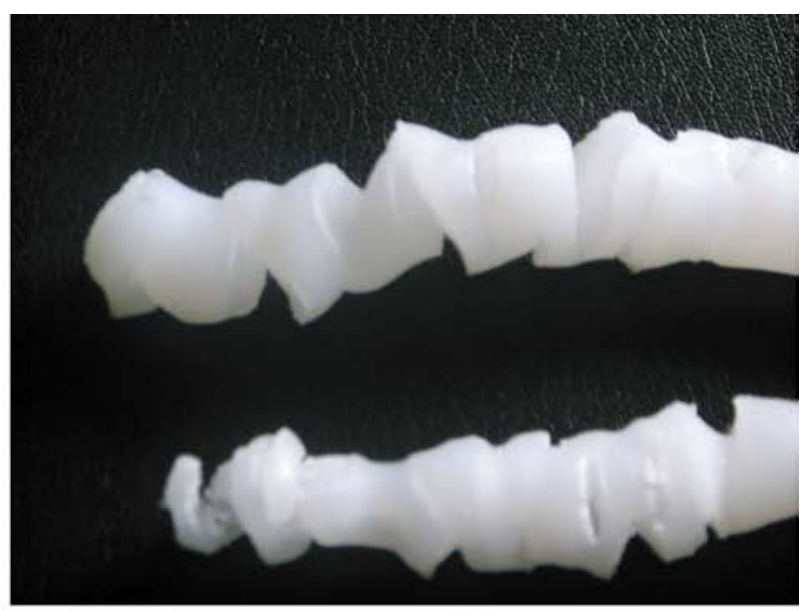

b) the temperature window $154-157^{\circ} \mathrm{C}$, yielding an 


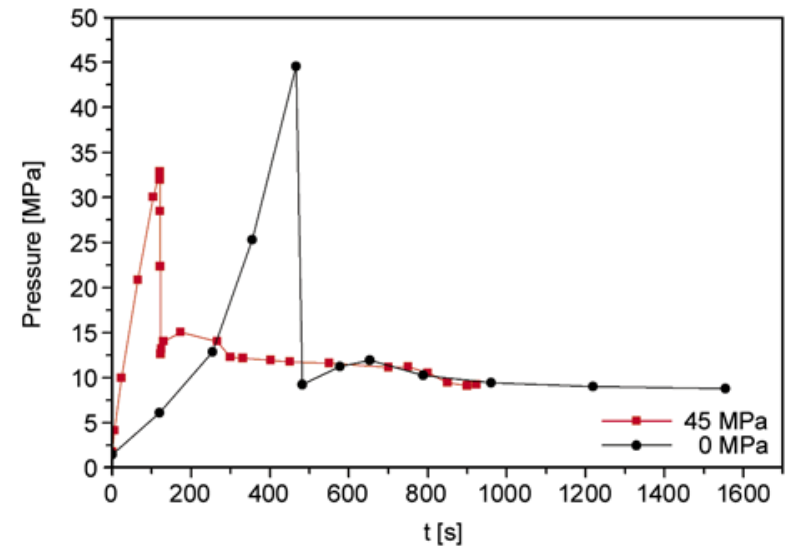

Figure 4. Time evolution of extrusion pressure profile under different annealing pressures during annealing at $200^{\circ} \mathrm{C}$ for $1 \mathrm{~h}\left(M_{\mathrm{W}}=3.5 \cdot 10^{6} \mathrm{~g} / \mathrm{mol} ; L / D=10: 5\right.$, $\mathrm{mm} / \mathrm{mm} ; v=0.035 \mathrm{~mm} / \mathrm{s} ; T=154^{\circ} \mathrm{C}$ )

$(30,60$, or $90 \mathrm{~min})$ under pressure (45 or $0 \mathrm{MPa})$. The melt temperature was then decreased to the temperature window and conditioned for $15 \mathrm{~min}$. The extrusion pressure evolution with time was recorded during extrusion at a piston velocity of $0.035 \mathrm{~mm} / \mathrm{s}$.

Figure 4 shows the effect of annealing pressure on the extrusion pressure. After being extruded for about $130 \mathrm{~s}$, the extrusion pressure drops for the melt annealed under pressure, whereas the pressure drop delays to over $450 \mathrm{~s}$ for the melt annealed without pressure. Annealing under pressure also decreases the maximum extrusion pressure by more than $10 \mathrm{MPa}$. This behavior indicates that the melt is loosely packed when annealed without pressure, but well-compacted under high pressure. This also explains the linear increase in the extrusion pressure during the initial stage in Figure 2. However, the temperature window effect always occurs whether annealing pressure is applied or not. The

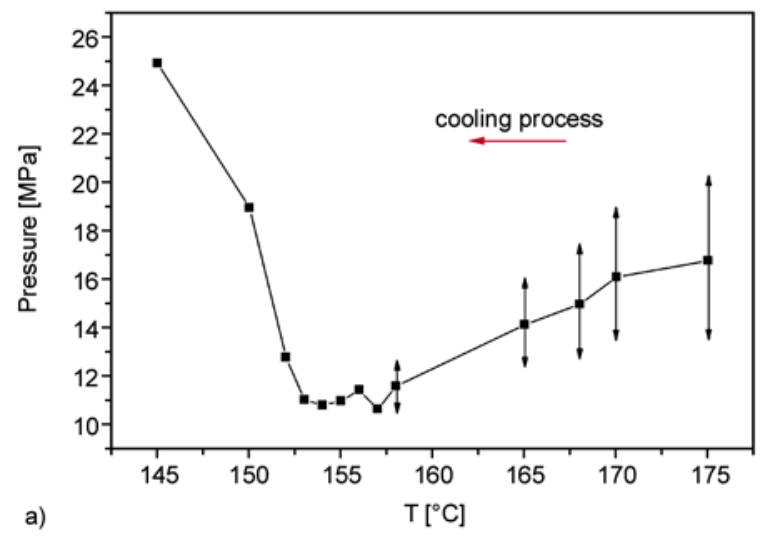

temperature window effect does not depend on the annealing process because the extrusion pressure always exhibits a linear increase with time in the initial stage and abruptly decreases at a maximum value, finally reaching a stable value. The only difference is the starting time of the temperature window effect and the strength of the pressure drop. Annealing under higher temperature, greater pressure, and longer time leads to a faster and larger extrusion pressure drop.

\subsection{Effects of heating/cooling reversibility}

To study the reversibility of the temperature window effect, we extruded UHMWPE during heating and cooling $\left(145 \leftrightarrow 175^{\circ} \mathrm{C}\right)$. UHMWPE was heated to $175^{\circ} \mathrm{C}$ and conditioned for $15 \mathrm{~min}$ before extrusion at a constant piston velocity to obtain the extrusion pressure. The extrusion pressure fluctuated with time; thus, the average pressure was recorded and the amplitudes were represented by arrows in Figure 5. The melt was cooled to a preset temperature and the process was repeated. A stable extrusion pressure was recorded in the temperature window $\left(154-157^{\circ} \mathrm{C}\right)$. Further cooling below the temperature window linearly increased the extrusion pressure with time; thus, the pressure at a given time after extrusion was used. The melt was then heated and the same extrusion and data recording procedures as those conducted in the cooling process were repeated. Finally, the extrusion pressure was plotted with temperature, depicted in Figure 5.

The temperature window effect appears during both cooling and heating processes. The temperature window range and the minimum pressure do not depend on cooling or heating. However, the extrusion pressure initially decreases and then increases

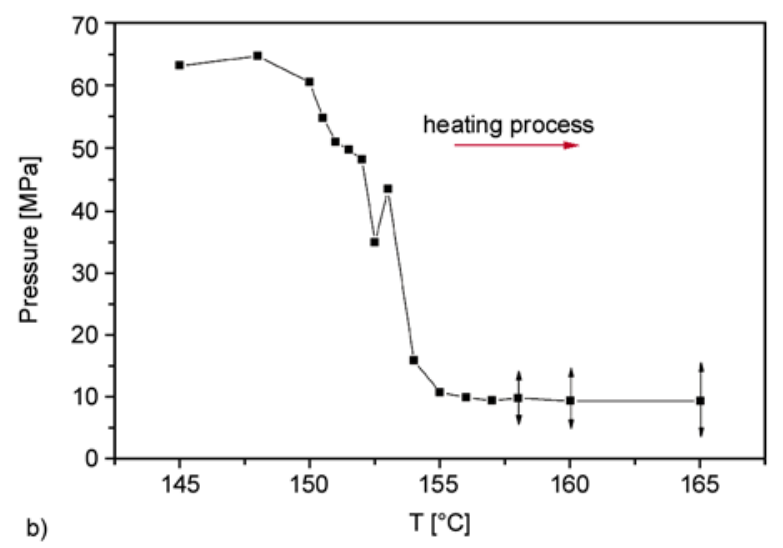

Figure 5. Extrusion pressure profile with extrusion temperature during a) cooling and b) heating $\left(M_{\mathrm{w}}=3.5 \cdot 10^{6} \mathrm{~g} / \mathrm{mol} ; L / D=\right.$ $10: 5, \mathrm{~mm} / \mathrm{mm} ; v=0.035 \mathrm{~mm} / \mathrm{s})$ 
with temperature upon cooling (Figure 5a), whereas it constantly decreases upon heating (Figure 5b). When extruded below the temperature window, the extrusion pressure during the heating process is higher than that during the cooling process at the same temperature. When extruded above the temperature window, the extrusion pressure during heating is lower than that during the cooling process at the same temperature. All these behaviors indicate that the most critical step in extrusion is to induce the temperature window effect, which depends on the stability of the oriented chain structure. The extrusion is a process to induce the chain orientation, while the temperature affects the stability of the oriented chain structure. The two factors combined together determine whether the temperature window effect happens or not. The extrusion pressure should be sufficiently high to induce the chain orientation, particularly during the heating process. The chain relaxation time is very long, so the oriented chain structure is maintained to some extent after the temperature window effect happens, leading to a relatively lower extrusion pressure. Therefore, the extrusion pressure at the same temperature is reversed during the cooling and heating processes. Kolnaar and Keller [11] reported that the window effect arises both upon heating and cooling in extruding MHMWPEs. However, the occurrence of the effect on cooling was erratic in their experiments. The window effect always appears upon heating but does not always appear upon cooling. Furthermore, if it does appear upon cooling, it is not always at the same strength [11]. This was eventually traced to a structure 'memory' effect [11], which implies that some structure formation can be induced more reliably in the heating than in the cooling process of a thermal cycle; once introduced, it can leave a structure memory in the melt state. Different from the extrusion of MHMWPE, the window effect always occurs both upon heating and cooling during UHMWPE extrusion. One possible reason is that the chain relaxation time of the UHMWPE chains is much longer so that the chain alignment induced by elongational flow can at least be partially maintained. In the second heating or cooling cycle, these well-aligned chains can re-induce the temperature window effect.

\subsection{Effects of piston velocity}

After being melted at high temperature, the UHMWPE melt was cooled to the temperature window. The temperature was kept constant, and the melt was extruded at a constant speed. After occurrence of the temperature window effect, the trace of extrusion pressure with time was recorded. The piston velocity was increased stepwise, and the corresponding pressure evolution with time was recorded. The temperature was increased, and the process was repeated to observe the extrusion pressure traces with time (Figure 6a-d). The final equilibrium pressure was recorded in stable extrusion, whereas average pressure was calculated in fluctuation. The variations in the extrusion pressure with piston velocity under different temperatures were plotted, and depicted in Figure 6e.

Figures $6 a-d$ show that the extrusion pressure is stable below a critical piston velocity. Outside this setting, it fluctuates with time. Higher piston velocity generates more severe fluctuation. The critical piston velocities decrease with the extrusion temperature, which are $0.20,0.10,0.05$, and $0.05 \mathrm{~mm} / \mathrm{s}$ at extrusion temperatures of $154,155,156$, and $157^{\circ} \mathrm{C}$, respectively. Figure $6 \mathrm{e}$ reveals the effects of piston velocity and extrusion temperature on extrusion pressure. Apparently, the extrusion pressure decreases with an increase in piston velocity and extrusion temperature. The extrusion pressure at $154^{\circ} \mathrm{C}$ is inconsistent with the trend possibly because of experimental errors.

The UHMWPE chains are aligned along the flow direction in the die during extrusion, and alignment becomes exceedingly complete with the increase in piston velocity, decreasing flow resistance. When the speed reaches a critical value, the wall slip and wall stick take place alternately. Thus, the extrudate is significantly distorted because of the melt fracture. With the increase in temperature in the temperature window, the thermal motion of the chains increases, which is favorable to the extension and alignment of the chains. This increase in the thermal motion of the chains decreases the extrusion pressure. At the same time, the stability maintaining the alignment of extended chains decreases; hence, the range of piston velocity for steady extrusion becomes narrow. 

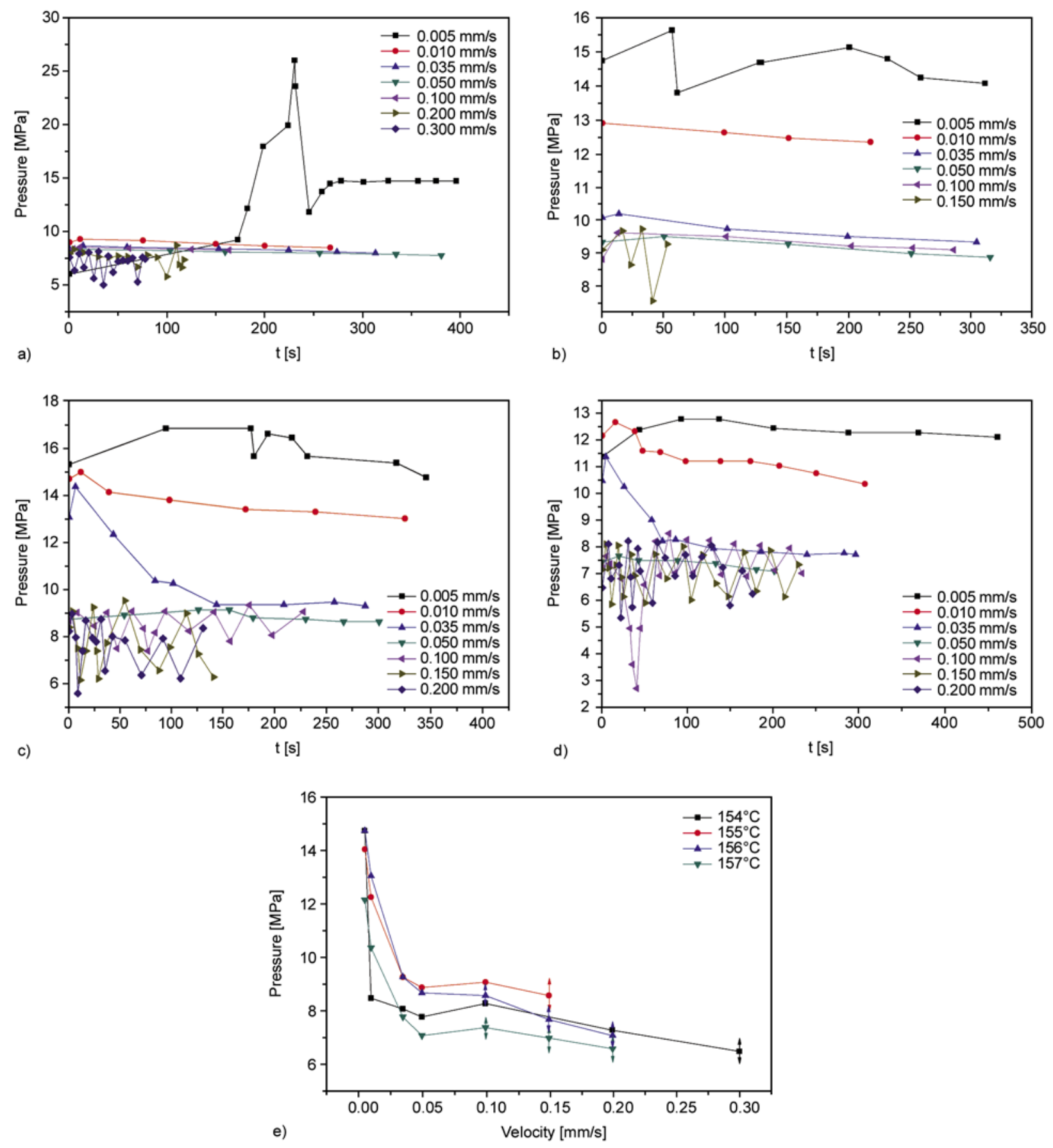

Figure 6. Extrusion pressure traces with time (a) $T=154^{\circ} \mathrm{C}$; b) $T=155^{\circ} \mathrm{C}$; c) $T=156^{\circ} \mathrm{C}$; d) $T=157^{\circ} \mathrm{C}$ ) and velocity e) $\left(M_{\mathrm{w}}=3.5 \cdot 10^{0} \mathrm{~g} / \mathrm{mol} ; L / D=10: 5, \mathrm{~mm} / \mathrm{mm}\right)$

\subsection{Effects of $L / D$ ratio of the die}

After being melted sufficiently, the UHMWPE melt was cooled and maintained at a preset temperature. The melt was extruded at a constant piston velocity, and the stable extrusion pressure was recorded. In unstable extrusion, the average pressure was calculated, and the fluctuation amplitude of pressure was represented by double arrows in Figure 7. With further decrease in temperature, the extrusion pressure was recorded at a given temperature until the melt could no longer be extruded. The procedure was repeated for the other two dies with different $L / D$ ratios. The extrusion pressure traces with temperature during extrusion using dies with different $L / D$ ratios are shown in Figure 7.

Figure 7 clearly reveals that the trend of extrusion pressure with temperature and the range of the temperature window effect do not depend on the $L / D$ 


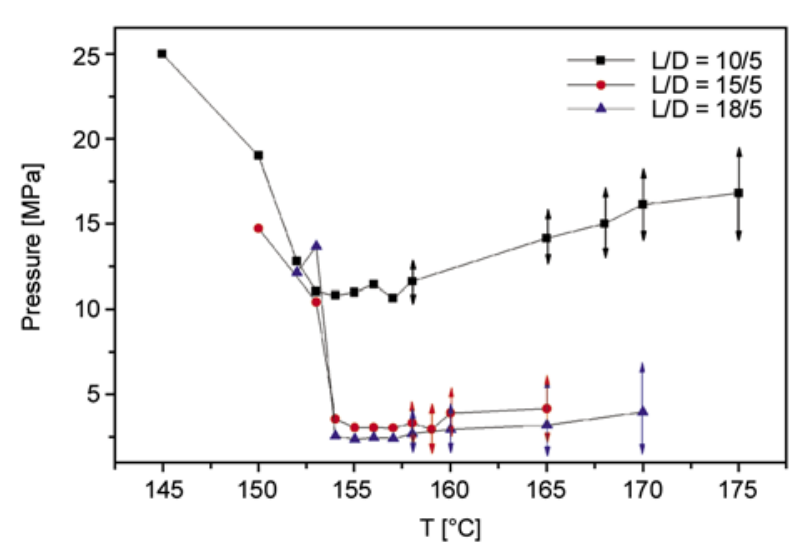

Figure 7. Effects of temperature on the extrusion pressure using extrusion dies with different $L / D$ ratios $\left(M_{\mathrm{w}}=3.5 \cdot 10^{6} \mathrm{~g} / \mathrm{mol} ; v=0.035 \mathrm{~mm} / \mathrm{s}\right)$

ratio of the die. In addition, the pressure decreases with the increase in $L / D$ ratio at the same temperature. The temperature window effect is attributed to the chain alignment at the convergent die entrance by elongational flow, indicating that the formation of a chain-extended structure and the stability of this structure are key factors. The die entry geometry and angle are unchanged. Accordingly, the elongational flow field at the entrance is also unchanged. Thus, the temperature window effect is not affected by $L / D$ ratio. The strength of pressure drop increases with $L / D$ ratio because of the relaxation of the chains in the capillary. The long chains are extended more sufficiently with the increase in capillary length, thereby generating a larger pressure drop.

\subsection{Effects of molecular weight}

We extruded three UHMWPE resins to study the effect of molecular weight on the temperature window effect. The extrusion pressure versus extrusion

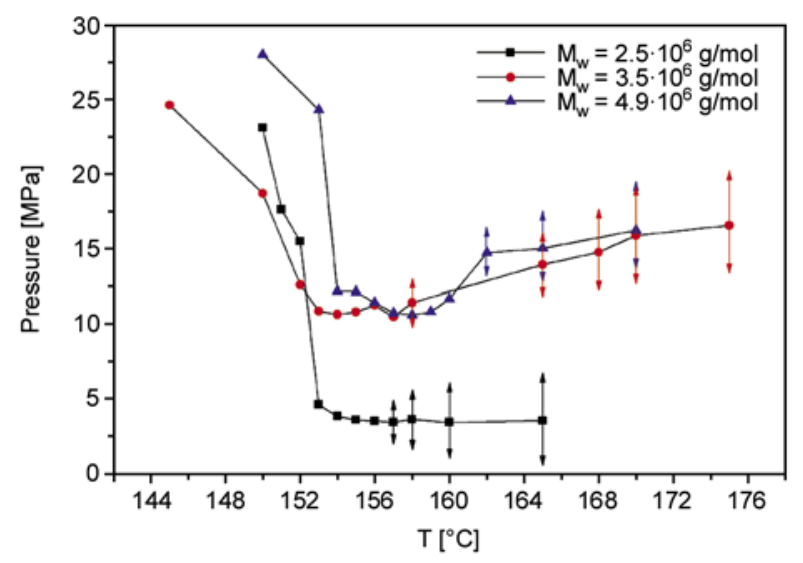

Figure 8. Effects of molecular weight on extrusion pressure at different extrusion temperatures $(L / D=10: 5$, $\mathrm{mm} / \mathrm{mm} ; v=0.035 \mathrm{~mm} / \mathrm{s}$ ) temperature traces for different molecular weights are shown in Figure 8.

The three types of UHMWPE all exhibit the temperature window effect during extrusion. Below the window, the extrusion pressure increases with time and blockage happens; above the window, the pressure fluctuates. In the window, the pressure significantly drops to a stable value, yielding a smooth extrudate surface. The ranges of the temperature window for UHMWPE with molecular weights of $2.5 \cdot 10^{6}, 3.5 \cdot 10^{6}$, and $4.9 \cdot 10^{6} \mathrm{~g} / \mathrm{mol}$ are $153-156$, $154-157$, and $157-160^{\circ} \mathrm{C}$, respectively. A quantitative relationship between the molecular weight and the mean temperature $(T)$ of the window effect is obtained by linear fitting of the data, which gives the Equation (1):

$$
\begin{aligned}
T \pm 1.5\left[{ }^{\circ} \mathrm{C}\right] & =1.7 \cdot 10^{-6}\left[{ }^{\circ} \mathrm{C} \cdot \mathrm{mol} / \mathrm{g}\right] \cdot M_{\mathrm{w}}[\mathrm{g} / \mathrm{mol}]+ \\
& +150\left[{ }^{\circ} \mathrm{C}\right]
\end{aligned}
$$

The onset extrusion temperature required to induce the temperature window effect increases with molecular weight. In fact, the temperature window observed in MHMWPE $\left(2.8 \cdot 10^{5}-4.0 \cdot 10^{5} \mathrm{~g} / \mathrm{mol}\right)$ by Kolnaar and Keller is $151 \pm 1^{\circ} \mathrm{C}$ [14], which also satisfies Equation (1) quite well. With the increase in molecular weight, the chain length increases and chain mobility decreases. The energy needed to activate chain extension and alignment increases; hence the onset temperature employed to induce the temperature window effect rises. In addition, the extrusion pressure in the temperature window also increases with the molecular weight of UHMWPE. This is due to the fact that the chain entanglement density increases with molecular weight, and the shear stress needed to disentangle the long chains during flowing in the die increases accordingly.

\subsection{Mechanism analysis}

The processability of UHMWPE is determined by the mobility of its macromolecules. In the melt state, long chains are entangled, and chain relaxation is slow. In the crystal state, long chains are folded or extended in an orderly manner with less entanglement. Under equilibrium conditions, the quiescent melting point of nascent UHMWPE is around $142-143^{\circ} \mathrm{C}$, very close to the theoretical equilibrium melting point of orthorhombic polyethylene crystals. After erasing the thermal history, the quiescent melting point decreases to $132-135^{\circ} \mathrm{C}$ 
[26]. In the flow state, the elongational flow in the die causes chain extension and induces the formation of extended chain crystals. Therefore, a mixture of melt phase and crystal like phase coexists at temperatures around $10^{\circ} \mathrm{C}$ higher than the theoretical quiescent melting point. UHMWPE continues to exhibit a solidified behavior with large elasticity, blocking the extrusion at the die entrance.

According to chain dynamics, phase and its behavior are closely related to material properties, and these properties may change significantly during phase transition [23]. There are three types of polyethylene crystal phases, i.e., orthorhombic, hexagonal, and monoclinic [30]. The hexagonal crystal phase has a chain-extended structure and a large laminar thickness, which demonstrates a higher chain mobility than in the other two phases [22]. However, the hexagonal crystal phase exists stably only under high temperature $\left(>230^{\circ} \mathrm{C}\right)$ and pressure (>360 MPa) during the equilibrium process [30]. These strict conditions are difficult to satisfy in practical processing. The extremely large size of UHMWPE chains determines its phase transition, which proceeds much more slowly than that in low molecular materials, making the equilibrium stable phases difficult to obtain. Thus, metastable phases may appear during the phase transition process.

The temperature window effect may be due to the formation of a metastable mobile phase induced by the elongational flow [31]. The chains are aligned along the extrusion direction in the die during extrusion and form a metastable structure similar to that of the hexagonal phase of polyethylene. The verification of this metastable phase transition needs specially designed equipment not readily available in our lab. However, many reports on studying the metastable phase transition of polyethylene can be found in literature.

Waddon and Keller [10] observed the metastable hexagonal phase transition in the temperature window of $150 \pm 1^{\circ} \mathrm{C}$ in MHMWPE extrusion $\left(2.8 \cdot 10^{5} \mathrm{~g} / \mathrm{mol}\right)$ by in situ XRD. Rastogi et al. [27] determined the metastable hexagonal phase transition of UHMWPE $\left(3 \cdot 10^{5}-5 \cdot 10^{5} \mathrm{~g} / \mathrm{mol}\right)$ at around $205^{\circ} \mathrm{C}$ by in situ XRD investigation with isobaric (160 and $180 \mathrm{MPa}$ ) heating. Chen et al. [32] examined the metastable phase transition of polyethylene $\left(7.8 \cdot 10^{4} \mathrm{~g} / \mathrm{mol}\right)$ under a transmission electron micro- scope. All these reports show that the metastable phase transition of polymers can be induced by elongational and shear flow in melts and mechanical deformation in solids at certain temperature and pressure.

The chain dynamics of UHMWPE during extrusion in the temperature window is complicated. From a mechanics point of view, the melt flow resistance, mainly including the stick force at wall and the shear force between chains, depends on the shear rate. When the pressure is high enough to overcome the flow resistance, the melt starts to flow and the chains are oriented along the flow direction. The oriented chain structure leads to significantly reduced flow resistance and its stability depends on the shear rate, or the piston velocity. From a thermodynamic point of view, flow induced chain extension results in the formation of extended-chain crystals; on the other hand, thermally induced chain relaxation results in the melting of crystals. The increased thermal mobility leads to faster relaxation because of entropic forces, which entangles the chains. The stability of the mesophase is, therefore, determined by the competition between external and internal factors.

Temperature is the most important factor in the temperature window effect. It should be high enough to activate chain extension, but sufficiently low to avoid chain entanglement. Therefore, it should be on the borderline between solid and melt. Because viscosity is proportional to the power 3.4 of molecular weight [11], the temperature needed to activate the rheological flow of UHMWPE melt should increase accordingly. This explains the onset temperature required to trigger the window effect increasing with the molecular weight of UHMWPE (Figure 8). Annealing and thermal history do not change the chain structure. Hence, they do not influence the temperature window effect. The piston velocity and $L / D$ ratio mainly affect the chain extension in the die. With the increase in piston velocity and $L / D$ ratio, the chain extension becomes exceedingly complete. However, if the velocity is excessively high, wall slip is prone to happen, especially at higher extrusion temperatures in which the extended chain structure is easily broken to form entanglements. This explains the results in Figure 6 and Figure 7. 


\section{Conclusions}

UHMWPE rods were ram extruded at low temperature and pressure using the temperature window effect. In the narrow temperature window, the extrusion pressure abruptly decreases, and the undistorted extrudates show a smooth surface. This effect is attributed to the formation of a transient mesophase induced by the elongational flow in the convergent capillary die, resulting in the alignment of chains along the flow direction. This phase exhibits an extended chain structure similar to the mobile hexagonal phase at high temperature and pressure under the equilibrium state. However, the phase is metastable and becomes stable under certain conditions. This study explored the factors influencing metastability, such as extrusion temperature, annealing process, thermal history, extrusion speed, $L / D$ ratio of the die, and molecular weight of UHMWPE. The conclusions are summarized as follows:

The temperature window is in the range $153-160^{\circ} \mathrm{C}$, and increases with the increase in molecular weight. The effect does not depend on the annealing process and is reversible on either cooling or heating. The extrusion pressure decreases with the increase in extrusion speed and temperature. It begins to fluctuate when the speed is greater than a critical value; the higher the temperature, the smaller the critical value. The effect does not depend on the $L / D$ ratio of capillary die, but the minimal pressure decreases with the increase in $L / D$ ratio. The minimal extrusion pressure in the temperature window increases with the increase in molecular weight.

The proposed method provides a new solution for the difficulties encountered in processing UHMWPE. Compared with other methods, it features no processing aids, no blending polymers, no equipment improvement, along with attractive attributes such as low energy consumption and high productivity.

\section{Acknowledgements}

This work was financially supported by the National Natural Science Foundation of China (No. 50803019) and the Guangdong Natural Science Fund (No. 8451064101000727), and partially supported by the Opening Project of The Key Laboratory of Polymer Processing Engineering, Ministry of Education, China.

\section{References}

[1] Dumitriu S.: Polymeric biomaterials. Marcel Dekker, New York (2002).
[2] Kelly J. M.: Ultra-high molecular weight polyethylene. Journal of Macromolecular Science Part C: Polymer Reviews, 42, 355-371 (2002).

DOI: $10.1081 / \mathrm{MC}-120006452$

[3] Kurtz S. M., Muratoglu O. K., Evans M., Edidin A. A.: Advances in the processing, sterilization, and crosslinking of ultra-high molecular weight polyethylene for total joint arthroplasty. Biomaterials, 20, 1659-1688 (1999).

DOI: $10.1016 / \mathrm{S} 0142-9612(99) 00053-8$

[4] Olley R. H., Hosier I. L., Bassett D. C., Smith N. G.: On morphology of consolidated UHMWPE resin in hip cups. Biomaterials, 20, 2037-2046 (1999).

DOI: $10.1016 /$ S0142-9612(99)00107-6

[5] Wunderlich B., Arakawa T.: Polyethylene crystallized from the melt under elevated pressure. Journal of Polymer Science Part A: General Papers, 2, 36973706 (1964).

DOI: $10.1002 /$ pol.1964.100020828

[6] Rees D. V., Bassett D. C.: Origin of extended-chain lamellae in polyethylene. Nature, 219, 368-370 (1968). DOI: $10.1038 / 219368 \mathrm{a} 0$

[7] Ivan'kova E. M., Myasnikova L. P., Marikhin V. A., Baulin A. A., Volchek B. Z.: On the memory effect in UHMWPE nascent powders. Journal of Macromolecular Science Part B: Physics, 40, 813-832 (2001). DOI: $10.1081 / \mathrm{MB}-100107563$

[8] Egorov V. M., Ivan'kova E. M., Marikhin V. A., Myasnikova L. P., Drews A.: On fine structure of nascent UHMWPE reactor powders. Journal of Macromolecular Science Part B: Physics, 41, 939-956 (2002).

DOI: $10.1081 / \mathrm{MB}-120013075$

[9] Uehara H., Kanamoto T., Kawaguchi A., Murakami S.: Real-time X-ray diffraction study on two-stage drawing of ultra-high molecular weight polyethylene reactor powder above the static melting temperature. Macromolecules, 29, 1540-1547 (1996).

DOI: $10.1021 / \mathrm{ma} 951222 \mathrm{y}$

[10] Waddon A. J., Keller A.: A temperature window of extrudability and reduced flow resistance in high-molecular-weight polyethylene; Interpretation in terms of flow-induced mobile hexagonal phase. Journal of Polymer Science Part B: Polymer Physics, 28, $1063-$ 1073 (1990). DOI: $10.1002 /$ polb.1990.090280706

[11] Kolnaar J. W. H., Keller A.: A temperature window of reduced flow resistance in polyethylene with implications for melt flow rheology: 1. The basic effect and principal parameters. Polymer, 35, 3863-3874 (1994). DOI: 10.1016/0032-3861(94)90269-0

[12] Kolnaar J. W. H., Keller A.: A temperature window of reduced flow resistance in polyethylene with implications for melt flow rheology: 2. Rheological investigations in the extrusion window. Polymer, 36, 821-836 (1995).

DOI: $10.1016 / 0032-3861(95) 93114-2$ 
[13] Kolnaar J. W. H., Keller A.: A temperature window of reduced flow resistance in polyethylene with implications for melt flow rheology: 3. Implications for flow instabilities and extrudate distortion. Polymer, 38, 1817-1833 (1997).

DOI: $10.1016 / \mathrm{S} 0032-3861(96) 00707-0$

[14] Kolnaar J. W. H., Keller A.: A singularity in the melt flow of polyethylene with wider implications for polymer melt flow rheology. Journal of Non-Newtonian Fluid Mechanics, 69, 71-98 (1997).

DOI: 10.1016/S0377-0257(97)00017-7

[15] Keller A., Hikosaka M., Rastogi S., Toda A., Barham P. J., Goldbeck-Wood G.: An approach to the formation and growth of new phases with application to polymer crystallization: Effect of finite size, metastability, and Ostwald's rule of stages. Journal of Materials Science, 29, 2579-2604 (1994).

DOI: $10.1007 / \mathrm{BF} 00356806$

[16] Keller A., Hikosaka M., Rastogi S., Toda A., Barham P. J., Goldbeck-Wood G., Windle A. H., Thomas E. L., Bassett D. C.: The size factor in phase transitions: Its role in polymer crystal formation and wider implications. Philosophical Transactions of the Royal Society of London, Series A, 348, 3-17 (1994).

DOI: $\underline{10.1098 / \text { rsta.1994.0077 }}$

[17] Keller A., Hikosaka M., Rastogi S.: The role of metastability in phase transformations: New pointers through polymer mesophases. Physica Scripta, 66, 243-247 (1996).

DOI: $10.1088 / 0031-8949 / 1996 / \mathrm{T} 66 / 045$

[18] Hikosaka M., Rastogi S., Keller A., Kawabata H.: Investigations on the crystallization of polyethylene under high pressure: Role of mobile phases, lamellar thickening growth, phase transformations, and morphology. Journal of Macromolecular Science Part B: Physics, 31, 87-131 (1992).

DOI: $10.1080 / 00222349208215459$

[19] Hikosaka M., Okada H., Toda A., Rastogi S., Keller A.: Dependence of the lamellar thickness of an extended-chain single crystal of polyethylene on the degree of supercooling and the pressure. Journal of the Chemical Society, Faraday Transactions, 91, 25732579 (1995).

DOI: 10.1039/FT9959102573

[20] Hikosaka M., Amano K., Rastogi S., Keller A.: Lamellar thickening growth of an extended chain single crystal of polyethylene. 1. Pointers to a new crystallization mechanism of polymers. Macromolecules, 30, 20672074 (1997).

DOI: $10.1021 / \mathrm{ma} 960746 \mathrm{a}$

[21] Hikosaka M., Amano K., Rastogi S., Keller A.: Lamellar thickening growth of an extended chain single crystal of polyethylene (II): $\Delta T$ dependence of lamellar thickening growth rate and comparison with lamellar thickening. Journal of Materials Science, 35, 51575168 (2000). DOI: 10.1023/A:1004804420369
[22] Rastogi S., Hikosaka M., Kawabata H., Keller A.: Role of mobile phases in the crystallization of polyethylene. Part 1. Metastability and lateral growth. Macromolecules, 24, 6384-6391 (1991).

DOI: $10.1021 / \mathrm{ma} 00024 \mathrm{a} 003$

[23] Cheng S. Z. D.: Phase transitions in polymers: The role of metastable states. Elsevier, Amsterdam (2008).

[24] Somani R. H., Yang L., Zhu L., Hsiao B. S.: Flowinduced shish-kebab precursor structures in entangled polymer melts. Polymer, 46, 8587-8623 (2005).

DOI: $10.1016 /$ j.polymer.2005.06.034

[25] van Bilsen H. M. M., Fischer H., Kolnaar J. W. H., Keller A.: A temperature window of reduced flow resistance in polyethylene: In situ WAXS. Macromolecules, 28, 8523-8527 (1995).

DOI: $10.1021 / \mathrm{ma} 00129 \mathrm{a} 009$

[26] Tashiro K., Sasaki S., Kobayashi M.: Structural investigation of orthorhombic-to-hexagonal phase transition in polyethylene crystal: The experimental confirmation of the conformationally disordered structure by $\mathrm{X}$-ray diffraction and infrared/raman spectroscopic measurements. Macromolecules, 29, 7460-7469 (1996). DOI: $10.1021 / \mathrm{ma960333+}$

[27] Rastogi S., Kurelec L., Lemstra P. J.: Chain mobility in polymer systems: On the borderline between solid and melt. 2. Crystal size influence in phase transition and sintering of ultrahigh molecular weight polyethylene via the mobile hexagonal phase. Macromolecules, 31, 5022-5031 (1998).

DOI: $10.1021 / \mathrm{ma9} 80261 \mathrm{~h}$

[28] Narh K. A., Keller A.: Differences in melt extrusion behaviour between ultra-high molecular weight polyethylene powder and a dried gel. Journal of Materials Science Letters, 10, 1301-1303 (1991).

DOI: $10.1007 / \mathrm{BF} 00722641$

[29] Rastogi S.: Role of metastable phases in polymer crystallization; Early stages of crystal growth. in 'Polymer crystallization: Observations, concepts, and interpretations' (eds. Reiter G., Sommer J-U.) Springer, Berlin, Vol 606, 17-47 (2003).

[30] Hikosaka M., Tsukijima K., Rastogi S., Keller A.: Equilibrium triple point pressure and pressure-temperature phase diagram of polyethylene. Polymer, 33, 2502-2507 (1992).

DOI: 10.1016/0032-3861(92)91130-T

[31] Chan C-K., Gao P.: A phenomenological model for the viscosity reductions in blends of HMMPE containing a small quantity of thermotropic liquid crystalline copolyester HBA/HQ/SA. Polymer, 46, 8151-8156 (2005). DOI: $10.1016 /$ j.polymer.2005.06.095

[32] Chen J-F., Yi L., Zhou Y-C., Zhou E-L.: High resolution transmission electron microscopy studies on nano-sized crystals of polyethylene and polypropylene (in Chinese). Chemical Journal of Chinese, 25, 966970 (2004). 